\title{
Summary of the First ISMRM-SNMMI Workshop on PET/MRI: Applications and Limitations
}

\author{
Thomas A. Hope ${ }^{1-3}$, Zahi A. Fayad ${ }^{4}$, Kathryn J. Fowler ${ }^{5}$, Dawn Holley ${ }^{6}$, Andrei Iagaru ${ }^{6}$, Alan B. McMillan ${ }^{7}$, \\ Patrick Veit-Haiback ${ }^{8}$, Robert J. Witte ${ }^{9}$, Greg Zaharchuk ${ }^{6}$, and Ciprian Catana ${ }^{10}$ \\ ${ }^{I}$ Department of Radiology and Biomedical Imaging, University of California San Francisco, San Francisco, California; ${ }^{2}$ Department \\ of Radiology, San Francisco VA Medical Center, San Francisco, California; ${ }^{3}$ UCSF Helen Diller Family Comprehensive Cancer \\ Center, University of California San Francisco, San Francisco, California; ${ }^{4}$ Translational and Molecular Imaging Institute, Icahn \\ School of Medicine at Mount Sinai, New York, New York; ${ }^{5}$ Department of Radiology, University of California San Diego, San Diego, \\ California; ${ }^{6}$ Department of Radiology, Stanford University Medical Center, Stanford, California; ${ }^{7}$ Department of Radiology, \\ University of Wisconsin-Madison, Madison, Wisconsin; ${ }^{8}$ Joint Department of Medical Imaging, Toronto General Hospital, University \\ Health Network, University of Toronto, Toronto, Canada; ${ }^{9}$ Department of Radiology, Mayo Clinic, Rochester, Minnesota; and \\ ${ }^{10}$ Athinoula A. Martinos Center for Biomedical Imaging, Department of Radiology, Massachusetts General Hospital, Harvard \\ Medical School, Charlestown, Massachusetts
}

Since the introduction of simultaneous PET/MRI in 2011, there have been significant advancements. In this review, we highlight several technical advancements that have been made primarily in attenuation and motion correction and discuss the status of multiple clinical applications using PET/MRI. This review is based on the experience at the first PET/MRI conference cosponsored by the International Society for Magnetic Resonance in Medicine and the Society of Nuclear Medicine and Molecular Imaging.

Key Words: PET/MRI; clinical applications, attenuation correction

J Nucl Med 2019; 60:1340-1346

DOI: 10.2967/jnumed.119.227231

$\mathbf{T}$ he first workshop on PET/MRI cosponsored by the International Society for Magnetic Resonance in Medicine (ISMRM) and the Society of Nuclear Medicine and Molecular Imaging (SNMMI) took place in Chicago on October 27-29, 2017. The goal of this conference was to provide a comprehensive discussion of PET/MRI spanning preclinical advances, novel pulse sequence development, clinical applications, and technologist training. During the 3-d meeting, there were 35 invited oral presentations, 54 poster presentations, and 14 oral abstract presentations.

\section{TECHNICAL HURDLES AND DEVELOPMENTS}

\section{Hardware Development}

Two devices that allow human whole-body simultaneous PET/ MRI are currently approved for clinical use - the Biograph mMR introduced in 2010 by Siemens Healthineers and the SIGNA timeof-flight system introduced in 2013 by GE Healthcare-whereas a

Received Feb. 8, 2019; revision accepted May 21, 2019.

For correspondence or reprints contact: Thomas A. Hope, University of California San Francisco, 505 Parnassus Ave., M-391, San Francisco, CA 94143.

E-mail: thomas.hope@ucsf.edu

Published online May 23, 2019.

COPYRIGHT (C) 2019 by the Society of Nuclear Medicine and Molecular Imaging. third one, the United Imaging UPMR790, was announced in 2017 by United Imaging Healthcare Co.

There are similarities and differences between the approaches chosen by the manufacturers for addressing the hardware challenges in integrating the two imaging modalities. On one hand, the MR components of these scanners all consist of 60-cm-bore 3-T superconducting magnets, whole-body gradient coil systems (44$50 \mathrm{mT} / \mathrm{m}$ amplitude, $200 \mathrm{~T} / \mathrm{m} / \mathrm{s}$ slew rate), and radiofrequency body coils. Additionally, the PET gantries have transaxial fields of view of approximately $60 \mathrm{~cm}$, the PET components are placed between the radiofrequency shield of the body coil and the gradient coils, and they use semiconductor-based photon detectors. On the other hand, there is more variability in the specific implementations of the PET components, from the photon detector technology (avalanche photodiodes vs. silicon photomultipliers) to the crystal size to the extent of the axial field of view (ranging from 25 to $33 \mathrm{~cm}$ ). The most significant difference between the available scanners is the availability of time-of-flight PET on two of the scanners.

Most importantly, minimal mutual interference and performance characteristics similar to stand-alone PET or PET/CT and MR scanners were reported by several groups $(1,2)$, demonstrating that the latest-generation integrated PET/MRI devices allow simultaneous acquisition of excellent-quality PET and MRI data, enabling the novel research and clinical applications discussed in the next sections.

\section{Attenuation Correction}

As expected, the performance of current MR-based attenuation correction (MRAC) techniques continues to be a matter of debate. Traditional approaches have typically ignored bone tissue, which leads to bias in reconstructed PET images on the order of $20 \%$ or more depending on the body region (3).

Until recently, two MRAC approaches have dominated the field. First, atlas-based approaches developed for both brain and wholebody applications have been shown to decrease PET bias to close to $5 \%$. However, they can perform suboptimally in subjects with substantial anatomic variations, are dependent on accurate imagebased registration, and can have region-specific bias in various regions (e.g., cerebellum). Second, approaches based on specialized 
MRI sequences using zero or ultrashort echo times have demonstrated efficacy, particularly in the brain (4) and pelvis (5). Although promising, methods using zero or ultrashort echo times have yet to be fully developed for whole-body MRAC, presumably because of technical challenges and lengthy scan times necessary to compensate for physiologic motion.

More recently, machine learning approaches using deep learning have demonstrated substantial potential for generating patientspecific attenuation maps with simple postprocessing based on conventionally acquired images (6). For example, in the brain, conventional T1-weighted images have been used to reduce PET bias to less than $2 \%(6)$. In the pelvis, deep learning MRAC approaches have demonstrated good performance using a variety of different input images, including those used for existing MRAC techniques (7), the use of hybrid Dixon and zero echo time approaches (8), and even conventional clinical images (9). Although whole-body techniques have yet to be demonstrated, it is expected that such capabilities will be achieved once the effect of physiologic motion on the MRI input images is minimized and subjects with abnormal anatomy are included in training and evaluation data sets.

It seems we are nearing a time where MRAC will no longer be discussed as an issue, at least in terms of its impact on the clinical interpretation of the images. However, an important point that needs to be considered is the relevance of MRAC for PET multicenter trials, as the standard phantoms used to validate the quantitative accuracy of PET/CT scanners overlook the issues associated with MRAC. Protocols need to be developed to allow for the incorporation of PET/MRI into multicenter trials. Although this task is in principle simpler in PET/MRI than in PET/CT, as there are only two devices available on the market, a complicating factor is that phantoms (ideally anthropomorphic) that consider both the CT and the MRI properties of the materials need to be developed to allow for validation of attenuation correction methodologies.

\section{Motion Correction}

Motion impacts all PET studies. Its negative effects include image blurring, quantification bias, artifacts due to attenuationemission data mismatches, and lesion misclassification and mispositioning. Substantial progress has recently been made in developing and applying MR-based approaches for head, respiratory, and cardiac motion characterization and compensation.

\section{NOTEWORTHY}

- Significant progress has been made in attenuation correction for integrated PET/MRI scanners, and the creation of accurate attenuation correction algorithms is possible with novel machine learning approaches.

- The translation of promising research applications is limited by the availability of radiotracers and reimbursement, particularly in neurooncology applications.

- Currently, benefits of simultaneity are limited to patient convenience and improved coregistration, the latter of which could be further improved using MR-assisted motion compensation.

- Preliminary consensus suggests advanced-level training is required to qualify technologists for performing PET/MRI examinations.

- Applications of PET/MRI at individual sites range widely and include prostate, neurodegeneration, and pancreatic cancer.
The rigid-body motion correction originally implemented for the Siemens BrainPET prototype and subsequently used in research studies (10) was recently demonstrated to have a positive impact on ${ }^{18} \mathrm{~F}$-FDG PET data quality and quantification in dementia patients (11).

In the case of respiratory motion compensation, the focus has slowly shifted toward developing methods that allow acquisition of the data needed to characterize motion efficiently (e.g., in $1 \mathrm{~min}$ (12)) or in the background of the sequences used for clinical purposes (13). As one example, the feasibility of performing concurrent reconstruction of respiratory motion-compensated dynamic contrast-enhanced MRI and PET data using motion vector fields derived from radial MR data by a compressed sensing approach was recently shown to produce better PET images both qualitatively and quantitatively, better fused PET and MR images, and dynamic contrast-enhanced MRI of diagnostic quality (14).

Substantial progress has also been made in the characterization of the cardiorespiratory motion of the heart. This was accomplished from free-breathing data acquired for diagnostic purposes over 5 min using a 3-dimensional golden radial phase encoding scheme $(15,16)$. Alternatively, if the MR data acquired in $90 \mathrm{~s}$ and a joint compressed sensing reconstruction and motion estimation are used to generate a cardiac and respiratory motion model, diagnostic MR sequences can be run throughout the rest of the PET acquisition (17).

Overall, the proof-of-principle PET motion compensation studies performed to date suggest that this interesting methodologic opportunity enabled by the simultaneous acquisition could have a substantial impact on research and clinical studies. The scanner manufacturers have now included relatively simple implementations of motion correction algorithms for brain and thoracoabdominal applications. It is likely that motion correction approaches will soon be implemented and incorporated into the clinical workflow, although there are further hurdles in the way of combined cardiac and respiratory motion correction that will need to be addressed.

\section{Imaging of Pulmonary Nodules}

Imaging the lung with MR continues to be challenging, and some investigators suggested that unenhanced CT of the chest should be performed immediately after PET/MRI. Instead, data were already presented suggesting that conventional MRI techniques such as motion-compensated T2-weighted images can detect clinically significant pulmonary nodules. Although MRI is likely to never equal CT for the detection of pulmonary nodules, the goal is to detect clinically significant nodules. Furthermore, short echo time sequences (ultrashort or zero echo time) offer the potential for pulmonary imaging that approaches the detection sensitivity of CT and may finally put to rest the need for concurrent $\mathrm{CT}$ of the chest $(18,19)$ so that lung imaging is not viewed as a barrier to the use of PET/MRI for oncologic applications.

\section{APPLICATIONS IN NEUROLOGY/NEUROONCOLOGY}

\section{Neurooncology}

Given the importance of MRI for characterization of intracranial neoplasms and the promise of novel radiotracers, PET/MRI should be a solution for imaging patients with intracranial neoplasms. With the development of standard reporting guidelines for amino acid PET, we are potentially getting closer to using these radiotracers in a clinical setting (20), although ${ }^{18} \mathrm{~F}$-FDG is still the only radiotracer available for clinical use in the United States. In looking toward Europe, the likely best radiotracer to take forward 
for neurooncology applications in the United States would be ${ }^{18} \mathrm{~F}$ fluoroethyltyrosine, although other tracers such as ${ }^{18} \mathrm{~F}$-fluciclovine or 3,4-dihydroxy-6- ${ }^{18}$ F-fluoro-L-phenylalanine may be available earlier.

The main promise of PET/MRI in neurooncology is the ability to differentiate between treatment-related changes after surgery or radiation and recurrent tumor. Early results have demonstrated that amino acid imaging using ${ }^{18} \mathrm{~F}$-fluoroethyltyrosine may improve the detection of recurrent glioma compared with MRI alone (21). A similar issue exists in evaluating intracranial metastatic lesions after radiation therapy, and amino acid PET/MRI may potentially help detect recurrent disease in this setting (22).

There are several settings in which PET/MRI may improve on MRI alone. One is in distinguishing low-grade from high-grade gliomas. Although there is promise in combining MRI and PET in this setting (23), biopsy is almost universally performed and so the role of imaging may be in evaluating the change in aggressiveness over time and determining when to repeat biopsy in patients with low-grade glioma. Overall, the pathologic characterization of glioma has changed dramatically since the introduction of the new World Health Organization schema, with several new molecularly defined subtypes. Although it is unlikely that imaging will be able to distinguish the various molecular subtypes, early work has already attempted to use PET/MRI to aid in lesion characterization (24). Treatment planning is another important role, and amino acid tracers appear to provide more information than is available on MRI and may improve radiotherapy plans (25).

Currently, the field is held back by a lack of consensus. Institutions use heterogeneous protocols (dynamic, single-time-point vs. dual-time-point PET) and interpretative criteria (tumor-to-background ratios vs. curve analysis). Multiinstitutional studies using harmonized prospective protocols for imaging and interpretation are needed to demonstrate that the results in the literature are in fact translatable to the clinic.

PET and MR images that are acquired sequentially may be fused accurately without the need for simultaneous PET/MRI. This capability may limit the adoption of PET/MRI in neurooncologic applications. Fusion can also be applied to epilepsy and neurodegenerative disease. Nonetheless, there is a benefit in terms of convenience for imaging patients in one session, and certain centers are using ${ }^{18} \mathrm{~F}$ FDG PET/MRI to combine whole-body PET imaging with brain MRI simply for the benefit of convenience.

\section{Epilepsy}

About a third of patients with epilepsy will be refractory to medical treatment, and approximately $50 \%$ of patients are categorized as having focal seizures and are potentially curable with surgery. In these patients, accurate localization of epileptogenic foci is critical for surgical planning. Focal cortical dysplasia is the most common etiology of epilepsy in children and can be difficult to detect on MRI, and ${ }^{18} \mathrm{~F}$-FDG PET coregistered to MRI has been shown to improve detection (26) and surgical outcome (27) in these patients.

Preliminary experience with hybrid PET/MRI has shown value in drug-refractory epilepsy patients presenting for potential surgery. Metabolic abnormalities and asymmetries compared with healthy controls have been demonstrated when the imaging has been supplemented with quantitative voxel-based analysis of the PET activity (28). The hybrid system has been shown to improve diagnostic yields in detecting potential epileptogenic lesions (29). Radiation dose reduction is an important benefit compared with PET/CT and MRI fusion in these patients, since they are typically children or young adults.
Beyond ${ }^{18} \mathrm{~F}-\mathrm{FDG}$, other PET radiotracers of relevance to epilepsy research would benefit from simultaneous PET and MRI acquisition. For example, a recent hybrid PET/MRI study using ${ }^{11} \mathrm{C}$-verapamil has shown uptake differences in drug-resistant epilepsy compared with drug responders and healthy controls (30). Central to epilepsy applications, optimization and standardization of attenuation correction are necessary for developing normative databases. These databases will enable accurate metabolic comparisons to healthy control populations.

\section{Neurodegeneration}

One of the early applications of PET/MRI has been to better understand dementia and other neurodegenerative diseases (31). Given that CT is largely insensitive to early Alzheimer disease changes, the availability of MRI is tremendously valuable. Although MRI is often obtained as a way to rule out suspected disease, the yield of these studies is overwhelmingly negative; a single examination therefore makes more sense from the cost-effectiveness perspective, besides being more convenient from the perspective of the patient, family, and researcher. Combination with the relatively longer MRI examination (30-45 $\mathrm{min}$ ) offers the potential to acquire a higher-quality PET scan at a normal dose or to reduce the dose without sacrificing image quality. Perfect coregistration and the potential to perform motion correction for both PET and MRI are also significant benefits (11).

In Alzheimer disease, several groups have suggested that dynamic amyloid PET/MRI could be valuable, with late images representating cortical amyloid burden whereas early images inform about cerebral blood flow, another important biomarker for Alzheimer disease. Although there are only limited reports of tau imaging using PET/ MRI (32), the improved soft-tissue contrast of MRI could also be helpful to better localize the precise regions of uptake for tau and to develop methods to avoid contamination of specific binding by offtarget binding in tau images.

PET/MRI for better assessment of Parkinson disease is also advancing. Investigational PET tracers such as ${ }^{18} \mathrm{~F}$-fluorinated $N-3$ fluoropropyl-2- $\beta$-carboxymethoxy-3- $\beta$-(4-iodophenyl)nortropane and ${ }^{11} \mathrm{C}$-PE2I may show more selectivity for the dopamine transporter. Still, there is limited information on the added value of PET/MRI using these tracers, although PET and MR motion correction strategies are likely even more relevant for patients with Parkinson disease, given the motor symptoms that accompany the disease, as well as new work identifying nigrosome-1 using susceptibility-weighted MRI (33).

Limitations impeding the more widespread use of PET/MRI for neurodegenerative disease include the use of PET/CT for legacy projects such as the Alzheimer's Disease Neuroimaging Initiative and the differences in attenuation correction between PET/CT and PET/MRI. However, the minimal differences in individual patients between attenuation correction performed by $\mathrm{CT}$ and attenuation correction performed by the latest-generation MRAC methods $(6,8)$ will hopefully lead to changes in the enrollment criteria for these large-scale projects. Furthermore, as more PET/MRI scanners are installed, consortiums such as the Dementias Platform U.K. (https://www.dementiasplatform.uk/) may play a larger role in collecting large data sets to help us understand whether simultaneity is helpful beyond convenience factors.

\section{CLINICAL APPLICATIONS IN ONCOLOGY}

\section{Prostate Cancer}

${ }^{11} \mathrm{C}$-choline has been approved by the Food and Drug Administration for use in prostate cancer since 2012; however, it is available at only a limited number of institutions and cannot be distributed. 
The Food and Drug Administration approval of ${ }^{18} \mathrm{~F}$-fluciclovine in 2016 brought a radiopharmaceutical able to detect prostate cancer at biochemical recurrence to the broader market, but the last 5 years witnessed a worldwide revolutionary adoption of ligands that bind to the prostate-specific membrane antigen (34). Additional classes of radiotracers are being evaluated, such as those targeting gastrinreleasing peptide receptors (35). With the availability and emergence of new radiotracers, the use of PET imaging in prostate cancer is growing.

The most obvious place for the use of PET/MRI in prostate cancer is during initial staging before definitive therapy. Although multiple groups have demonstrated that combined PET/MRI with ${ }^{18} \mathrm{~F}$-fluorcholine, ${ }^{18} \mathrm{~F}$-fluciclovine, and ${ }^{68} \mathrm{Ga}$-prostate-specific membrane antigen-11 may help in the detection of intermediate- to high-risk prostate cancer (36-39), the role of PET/MRI in the active-surveillance population for biopsy guidance has not yet been evaluated.

Although there are studies using PET/MRI to evaluate patients with suspected biochemical recurrence, the advances in PET/CT technology lead to fast, sensitive examinations that may limit the need for PET/MRI in this clinical scenario. Therefore, it is not immediately evident what the benefit of PET/MRI is over PET/CT for the detection of metastatic disease. In reference to local recurrence, a few studies have evaluated PET/MRI and shown small improvements over PET/CT, particularly the added value of dynamic contrast-enhanced imaging for the detection of local recurrence (40).

What may be of greater interest is the detection and characterization of bone lesions. Further work also needs to be performed to better understand the complementary role of diffusion-weighted MRI and PET imaging for assessment of response to treatment.

\section{Pancreatic Cancer}

PET and MRI are frequently used in pancreatic cancer for disease detection, lesion characterization, and staging, as well as for evaluation of recurrent disease and therapy monitoring (41). In pancreatic neuroendocrine tumor, PET/MRI using somatostatin receptor analogs (e.g., ${ }^{68}$ Ga-DOTATOC and ${ }^{68}$ Ga-DOTATATE) can improve the detection of liver metastases, particularly when hepatobiliary-phase imaging is used $(42,43)$. For adenocarcinoma, PET/MRI is equivalent to PET/CT for determining resectability and staging tumors (44). More unique is that the combination of diffusion-weighted imaging and metabolic markers (metabolic tumor volume) appears to predict advanced-stage tumor and correlates with progression-free survival in the adenocarcinoma population (45). Further work has shown that the ratio of total lesion glycolysis to peak enhancement from dynamic contrast-enhanced MRI is associated with poor overall survival (46). These data underline the need for multiparametric evaluation and research in hybrid imaging.

\section{Pediatric Applications}

One important application for PET/MRI is the imaging of oncologic pediatric patients. The main benefit in this setting is the decreased radiation dose from removing the CT component from PET/CT. CT imaging accounts for roughly $50 \%$ of the radiation dose, and therefore PET/MRI immediately reduces this dose (47). Additionally, the administered dose may be substantially reduced using MR information simultaneously obtained with artificial intelligence techniques such as deep learning (48). These approaches that decrease administered dose will be particularly relevant in the pediatric patient population, for which concerns over radiation dose are more pronounced. Workflow issues related to the increased need for anesthesia and sedation for PET/MRI compared with PET/CT still limit the applicability of PET/MRI, particularly in younger patients.

\section{Gynecologic Malignancy}

The benefits of combining PET and MRI in gynecologic malignancy were first demonstrated through retrospective fusion studies combining separately acquired data to improve staging accuracy (49).

The initial investigations looking at simultaneously acquired PET/MRI for gynecologic cancer demonstrated noninferiority over traditional PET/CT and MRI for staging and defining tumor volumes (50). More recent studies aimed to investigate potential unique advantages of the hybrid systems by focusing on multiparametric treatment response indicators (51), the impact on management decisions (52), the potential of multiparametric prognostic biomarkers (53), the use of novel tracers (54), and the correlation between PET and MRI parameters to facilitate a voxel-by-voxel approach (55). To date, it seems clear that clinical benefits derived individually from PET and MRI for diagnostic staging of cervical cancer are maintained in simultaneous PET/MRI, with the added benefit of improved imaging efficiency.

\section{CARDIOVASCULAR APPLICATIONS}

MRI and PET provide complementary information of clinical importance in cardiovascular applications, and in many cases, patients will undergo both examinations separately. In cardiac imaging, the ability to correct for the substantial cardiorespiratory motion in the PET data $(15,16,56)$ will be potentially significant in optimizing the detection and quantification of subtle uptake in the myocardium, such as in cardiac sarcoidosis, and in small structures such as the coronary arteries.

\section{Myocardial PET/MRI}

One of the most exciting potential clinical applications of PET/ MRI is the assessment of cardiac sarcoidosis. MRI allows for evaluation of myocardial structure, function, and pattern of injury on late gadolinium enhancement, whereas ${ }^{18} \mathrm{~F}$-FDG PET informs about myocardial and extracardiac inflammation via the elevated uptake of ${ }^{18}$ F-FDG by macrophages. The ability to coregister subtle uptake on both late gadolinium enhancement and PET improves disease detection and discrimination of active over inactive disease $(57,58)$.

In evaluating myocarditis, MRI is already widely used to confirm the diagnosis and rule out myocardial infarction based on the characteristic pattern of mid-wall late gadolinium enhancement. Simultaneously determining the activity of disease with ${ }^{18} \mathrm{~F}$ FDG PET (58) is also of potential interest for clinical management of myocarditis. Cardiac amyloidosis is another area in which PET data significantly complement MRI data. Characteristic late gadolinium enhancement can be used to diagnose amyloidosis but cannot differentiate between the two predominant forms of amyloid-acquired monoclonal immunoglobulin light-chain and transthyretin-related amyloid—which is important for determining prognosis. Recently it has been demonstrated that, similar to SPECT, patients with transthyretin-related amyloid exhibited increased myocardial activity of the PET bone tracer ${ }^{18} \mathrm{~F}$-fluoride $(59,60)$. In ischemic heart disease, the combination of MRI and PET measures of viability, function, and perfusion has also been investigated (61).

\section{Vascular PET/MRI}

Imaging inflammation in atherosclerotic plaque by ${ }^{18} \mathrm{~F}-\mathrm{FDG}$ PET has emerged as a major application of PET/MRI. Many studies have 
investigated plaque imaging in the large arteries (carotid artery, aorta, femoral artery) (62). Use of ${ }^{18} \mathrm{~F}$-sodium fluoride to investigate microcalcification has allowed investigation of coronary artery disease with PET/MRI $(63,64)$ and of aortic stenosis $(65)$.

\section{ADVANCES IN WORKFLOW}

When PET/MRI was first introduced commercially, it was directly compared with PET/CT in whole-body oncologic imaging and found to be more time-consuming because of the lengthy MR protocols (66). Since then, several shortened organ-specific protocols have been evaluated and implemented, and all showed diagnostic accuracy and lesion detectability comparable to either PET/CT or PET/MRI with fully diagnostic MR-protocols $(67,68)$.

Beyond MRI advances, new PET-detector technologies allowing for significantly reduced amounts of injected radiotracer offer considerably improved flexibility in acquisition time versus radiation burden $(69,70)$. Sah et al., for example, demonstrated that the amount of injected radiotracer can be reduced to $5 \%-10 \%$ of the currently clinically accepted standard dose while maintaining image quality (71). However, that study evaluated breast lesions with favorably low background on PET. The results in, for example, liver imaging (an organ with high background activity on ${ }^{18} \mathrm{~F}-\mathrm{FDG}$ PET imaging) may be different in terms of possible dose reduction. Also, such a reduction applies only to single-station imaging. However, the results nevertheless demonstrate that such improvements may offer new flexibility in PET/MRI workflow design and that single-station imaging (in comparison to standard whole-body imaging) will be a viable option for PET/MRI.

\section{THE BENEFITS OF SIMULTANEITY AND MACHINE LEARNING}

To date there is no clinical application of PET/MRI that truly leverages the simultaneous acquisition of PET and MRI, save for the associated increase in patient convenience, although certain applications such as vascular imaging may benefit from intrinsic coregistration more than others. Typical image interpretation evaluates the PET and MR images sequentially and then cognitively associates the findings to generate a final interpretation. Data on pancreatic cancer were presented in which combined interpretation of the apparent diffusion coefficient and uptake on ${ }^{18} \mathrm{~F}-\mathrm{FDG}$ PET helped predict clinical stage and progression-free survival (45), but that work, like many others, relied on manual regionof-interest measurements. As we move toward combined imaging biomarkers, there is immense potential for using machine learning to aid in interpretation of combined multiparametric PET and MRI data in ways that have not been feasible. Machine learning approaches have proven successful in the realm of MRAC. A machine learning approach may finally allow our community to demonstrate the benefit of simultaneous PET and MRI.

\section{LESSONS ON COLLABORATION}

Without well-defined hypotheses, established reference standards, and defined clinical protocols, pooling of highly variable clinical imaging data is unlikely to lead to useful information that can help increase the adoption of PET/MRI or develop new clinical indications. Large multicenter and multivendor data sets will be required to allow machine learning to achieve its full potential, and attention must be paid to harmonization of reference standards for valid training of the data (e.g., segmented pathology or clinical outcomes). The expense of these annotated data sets will likely prevent progress and straightforward data sharing. A simple first step toward collaboration is to standardize MRI protocols for specific clinical applications across sites to allow for future data collection.

\section{TECHNOLOGIST TRAINING}

As PET/MRI research and clinical applications continue to emerge and evolve, there remains the question of who is qualified to perform scanning. Would it be hybrid-PET/MRI technologists or, instead, PET technologists and MRI technologists working together? Ideally there would be an abundance of dual-trained technologists fluent in the unique aspects of PET and MRI. However, training opportunities are limited, time-intensive, and money-intensive, and the requirements are often confusing. There are currently no formal requirements or recommendations from either the SNMMI Technologist Section or the Society for MR Radiographers and Technologists regarding the qualifications to operate a PET/MRI scanner, although the preliminary consensus is that advanced-level education will be required (72).

Currently, there are 4 options to become certified in both nuclear medicine and MRI. The first is for a certified nuclear medicine technologist (NMT) to cross-train in MRI safety and procedures without obtaining certification. Although preferred, it is not currently a requirement to be certified in MRI to perform the duties of an MRI technologist. As such, it is possible for an NMT to crosstrain in MRI, learn MRI safety and applicable scan procedures, and operate a PET/MRI scanner. This is not ideal because the depth of education required to learn MRI safety, physics, instrumentation, sequence parameters, and image quality is significant. The second option is for an MRI technologist to become a certified NMT. This is the most challenging pathway because there is no postprimary path for a radiologic technologist to become an NMT. The MRI technologist would need to complete a nuclear medicine accredited program and take either the American Registry of Radiologic Technologists (ARRT) nuclear medicine examination or the Nuclear Medicine Technologist Certification Board examination. The third option is for an NMT to become certified in MRI. This is a postprimary pathway and therefore the easiest option to becoming dualcertified in NMT and MRI. An NMT with either an ARRT(N) or a Nuclear Medicine Technologist Certification Board certificate can qualify to take the ARRT or American Registry of MRI Technologists (ARMRIT) examination and become certified in MRI. The ARRT requires $16 \mathrm{~h}$ of structured education, documented performance of 125 MRI procedures, and passing of the ARRT examination. ARMRIT requires $1,000 \mathrm{~h}$ of MRI clinical experience and the passing of the ARMRIT examination. ARRT and ARMRIT training is often provided through accredited programs, which involve 9-12 mo of full-time training. A fourth option is for an MRI technologist to obtain a PET/CT certificate rather than become an NMT. In this path, MRI technologists with ARRT certification need to complete 700 documented hours of PET imaging, including radiopharmaceutical handling, injection, and imaging. It also requires completing $45 \mathrm{~h}$ of course work and passing the Nuclear Medicine Technologist Certification Board PET examination.

Although there currently does not exist a certification for PET/ MRI, this is a goal within the SNMMI Technologist Section and Society for MR Radiographers and Technologists educational community. For now, an NMT can pursue a postprimary MRI certification by qualifying to sit for either the ARRT or the ARMRIT examination. Obtaining dual certification in both NMT and MRI merely provides the qualifications to operate a PET/MRI scanner but does not fully address the unique aspects of this imaging 
modality. The lack of clarity and difficulty in training both in MRI and in PET have led to a dearth of appropriately trained technologists, and many institutions have relied on the use of two technologists (one trained in MRI and another in PET) to operate PET/ MRI scanners. This solution is less than ideal, and it is hoped that improvements in training requirements can be implemented.

\section{DRIVERS TO CLINICAL SUCCESS}

Unique to the ISMRM-SNMMI conference was a focus on clinical implementation of PET/MRI, and several practices presented perceived hurdles and potential solutions. There were two interesting observations across sites. First, each site highlighted that it had needed leaders at that site to develop a referral base. Typically, developing such leaders had involved not only participation on tumor boards but also personal communication of results to referring clinicians. Second, several sites indicated that allowing for an introductory period in which PET/MRI studies were made available free of charge had helped radiologists and referring clinicians become comfortable with the benefits of simultaneous imaging, which further helped cement future referral patterns and set the foundation for success.

Surprisingly there is large variation among PET/MRI practices. At two sites, pancreatic PET/MRI was the most common study, at another dementia PET/MRI was, whereas at a fourth it was prostate PET/MRI. This wide variability of clinical applications across sites highlights the benefit of frequent interactions to learn from one another about potential beneficial applications.

Physician training is also a hurdle in the way of more widespread implementation of PET/MRI. Societies have put forth recommendations on requirements for PET/MRI physician training (73). The development of the joint nuclear medicine and radiology training pathway may help increase the number of dual-trained physicians capable of interpreting studies (74). There are no standard approaches across institutions to reading PET/MRI scans; interpretation can involve either a single dual-trained reader or separate radiology and nuclear medicine readers.

An additional challenge identified by the group was fear of nonreimbursement, which prevented sites from performing studies for some indications. For example, multiple sites did not perform dementia imaging on PET/MRI because of the belief that a negative result on MRI had to be obtained before a dementia PET study could be performed. One site has been successful in performing dementia PET/MRI without the need for an antecedent MRI study. The same was true for the IDEAS trial (NCT02420756): technically, this trial requires certification of the PET scanner, and so it was believed that the studies cannot be performed on a PET/MRI scanner, but one site was successful in using its integrated scanner for imaging patients enrolled in this trial.

\section{CONCLUSION}

Although simultaneous PET/MRI has been a clinical reality for 8 years, there are numerous opportunities to further advance the field. Many of the initial technical developments focused on improvements in attenuation and motion correction. Soon, issues related to attenuation correction will be solved. Future directions should focus on applications in which simultaneous acquisition can lead to improved tissue characterization.

\section{DISCLOSURE}

Thomas Hope was supported by the Prostate Cancer Foundation (2017 Jonathan Kovler Young Investigator Award) and the National
Institutes of Health (grant R01CA212148). Zahi Fayad was supported by the National Institutes of Health (P01HL131478, R01HL071021, R01HL128056, R01HL144072, R01HL135878, and R01HL143814). Alan McMillan was supported by the National Institutes of Health (R01EB026708). Thomas Hope, Andrei Iagaru, Alan McMillan, and Greg Zaharchuk have received grant funding from GE Healthcare. The A.A. Martinos Center receives support for instrumentation from Siemens in the domains of MRI and PET/MRI. No other potential conflict of interest relevant to this article was reported.

\section{REFERENCES}

1. Karlberg AM, Sæther O, Eikenes L, Goa PE. Quantitative comparison of PET performance: Siemens Biograph mCT and mMR. EJNMMI Phys. 2016;3:5.

2. Levin CS, Maramraju SH, Khalighi MM, Deller TW, Delso G, Jansen F. Design features and mutual compatibility studies of the time-of-flight PET capable GE SIGNA PET/MR system. IEEE Trans Med Imaging. 2016;35:1907-1914.

3. Samarin A, Burger C, Wollenweber SD, et al. PET/MR imaging of bone lesions: implications for PET quantification from imperfect attenuation correction. Eur J Nucl Med Mol Imaging. 2012;39:1154-1160.

4. Ladefoged CN, Law I, Anazodo U, et al. A multi-centre evaluation of eleven clinically feasible brain PET/MRI attenuation correction techniques using a large cohort of patients. Neuroimage. 2017;147:346-359.

5. Leynes AP, Yang J, Shanbhag DD, et al. Hybrid ZTE/Dixon MR-based attenuation correction for quantitative uptake estimation of pelvic lesions in PET/MRI. Med Phys. 2017;44:902-913.

6. Liu F, Jang H, Kijowski R, Bradshaw T, McMillan AB. Deep learning MR imagingbased attenuation correction for PET/MR imaging. Radiology. 2018;286:676-684.

7. Torrado-Carvajal A, Vera-Olmos J, Izquierdo-Garcia D, et al. Dixon-VIBE deep learning (DIVIDE) pseudo-CT synthesis for pelvis PET/MR attenuation correction. J Nucl Med. 2019;60:429-435.

8. Leynes AP, Yang J, Wiesinger F, et al. Zero-echo-time and Dixon deep pseudoCT (ZeDD CT): direct generation of pseudo-CT images for pelvic PET/MRI attenuation correction using deep convolutional neural networks with multiparametric MRI. J Nucl Med. 2018;59:852-858.

9. Bradshaw TJ, Zhao G, Jang H, Liu F, McMillan AB. Feasibility of deep learningbased PET/MR attenuation correction in the pelvis using only diagnostic MR images. Tomography. 2018;4:138-147.

10. Roffman JL, Tanner AS, Eryilmaz H, et al. Dopamine D1 signaling organizes network dynamics underlying working memory. Sci Adv. 2016;2:e1501672.

11. Chen KT, Salcedo S, Chonde DB, et al. MR-assisted PET motion correction in simultaneous PET/MRI studies of dementia subjects. J Magn Reson Imaging. 2018; 48:1288-1296.

12. Manber R, Thielemans K, Hutton BF, et al. Practical PET respiratory motion correction in clinical PET/MR. J Nucl Med. 2015;56:890-896.

13. Munoz C, Neji R, Cruz G, et al. Motion-corrected simultaneous cardiac positron emission tomography and coronary MR angiography with high acquisition efficiency. Magn Reson Med. 2018;79:339-350.

14. Catalano OA, Umutlu L, Fuin N, et al. Comparison of the clinical performance of upper abdominal PET/DCE-MRI with and without concurrent respiratory motion correction (MoCo). Eur J Nucl Med Mol Imaging. 2018;45:2147-2154.

15. Kolbitsch C, Ahlman MA, Davies-Venn C, et al. Cardiac and respiratory motion correction for simultaneous cardiac PET/MR. J Nucl Med. 2017;58:846-852.

16. Robson PM, Trivieri M, Karakatsanis NA, et al. Correction of respiratory and cardiac motion in cardiac PET/MR using MR-based motion modeling. Phys Med Biol. 2018;63:225011.

17. Küstner T, Schwartz M, Martirosian P, et al. MR-based respiratory and cardiac motion correction for PET imaging. Med Image Anal. 2017;42:129-144.

18. Burris NS, Johnson KM, Larson PEZ, et al. Detection of small pulmonary nodules with ultrashort echo time sequences in oncology patients by using a PET/MR system. Radiology. 2016;278:239-246.

19. Jiang W, Ong F, Johnson KM, et al. Motion robust high resolution 3D freebreathing pulmonary MRI using dynamic 3D image self-navigator. Magn Reson Med. 2018;79:2954-2967.

20. Law I, Albert NL, Arbizu J, et al. Joint EANM/EANO/RANO practice guidelines/ SNMMI procedure standards for imaging of gliomas using PET with radiolabelled amino acids and $\left[{ }^{18}\right.$ F]FDG: version 1.0. Eur J Nucl Med Mol Imaging. 2019;46:540-557.

21. Pyka T, Hiob D, Preibisch C, et al. Diagnosis of glioma recurrence using multiparametric dynamic ${ }^{18}$ F-fluoroethyl-tyrosine PET-MRI. Eur J Radiol. 2018;103:32-37.

22. Lohmann P, Kocher M, Ceccon G, et al. Combined FET PET/MRI radiomics differentiates radiation injury from recurrent brain metastasis. Neuroimage Clin. 2018;20:537-542. 
23. Verger A, Filss CP, Lohmann P, et al. Comparison of ${ }^{18}$ F-FET PET and perfusion-weighted MRI for glioma grading: a hybrid PET/MR study. Eur J Nucl Med Mol Imaging. 2017;44:2257-2265.

24. Vettermann FJ, Felsberg J, Reifenberger G, et al. Characterization of diffuse gliomas with histone H3-G34 mutation by MRI and dynamic ${ }^{18}$ F-FET PET. Clin Nucl Med. 2018;43:895-898.

25. Harat M, Małkowski B, Makarewicz R. Pre-irradiation tumour volumes defined by MRI and dual time-point FET-PET for the prediction of glioblastoma multiforme recurrence: a prospective study. Radiother Oncol. 2016;120:241-247.

26. Salamon N, Kung J, Shaw SJ, et al. FDG-PET/MRI coregistration improves detection of cortical dysplasia in patients with epilepsy. Neurology. 2008;71:1594-1601.

27. Chassoux F, Rodrigo S, Semah F, et al. FDG-PET improves surgical outcome in negative MRI Taylor-type focal cortical dysplasias. Neurology. 2010;75:2168-2175.

28. Ding Y-S, Chen B-B, Glielmi C, Friedman K, Devinsky O. A pilot study in epilepsy patients using simultaneous PET/MR. Am J Nucl Med Mol Imaging. 2014;4:459-470.

29. Shin HW, Jewells V, Sheikh A, et al. Initial experience in hybrid PET-MRI for evaluation of refractory focal onset epilepsy. Seizure. 2015;31:1-4.

30. Shin JW, Chu K, Shin SA, et al. Clinical applications of simultaneous PET/MR imaging using (R)-[ $\left[{ }^{11} \mathrm{C}\right]$-verapamil with cyclosporin A: preliminary results on a surrogate marker of drug-resistant epilepsy. Am J Neuroradiol. 2016;37:600-606.

31. Drzezga A, Barthel H, Minoshima S, Sabri O. Potential clinical applications of PET/ MR imaging in neurodegenerative diseases. J Nucl Med. 2014;55(suppl):47S-55S.

32. Zhou Y, Bai B. Tau and Pet/Mri imaging biomarkers for detecting and diagnosing early dementia. Jacobs J Med Diagn Med Imaging. 2017;2:017.

33. Schwarz ST, Afzal M, Morgan PS, Bajaj N, Gowland PA, Auer DP. The 'swallow tail' appearance of the healthy nigrosome: a new accurate test of Parkinson's disease: a casecontrol and retrospective cross-sectional MRI study at 3T. PLoS One. 2014;9:e93814.

34. Afshar-Oromieh A, Babich JW, Kratochwil C, et al. The rise of PSMA ligands for diagnosis and therapy of prostate cancer. J Nucl Med. 2016;57(suppl):79S-89S.

35. Minamimoto R, Sonni I, Hancock S, et al. Prospective evaluation of ${ }^{68} \mathrm{Ga}-\mathrm{RM} 2$ PET/MRI in patients with biochemical recurrence of prostate cancer and negative findings on conventional imaging. J Nucl Med. 2018;59:803-808.

36. Park SY, Zacharias C, Harrison C, et al. Gallium 68 PSMA-11 PET/MR imaging in patients with intermediate- or high-risk prostate cancer. Radiology. 2018;288:495-505.

37. Eiber M, Weirich G, Holzapfel K, et al. Simultaneous ${ }^{68}$ Ga-PSMA HBED-CC PET/MRI improves the localization of primary prostate cancer. Eur Urol. 2016;70:829-836.

38. Kim Y-I, Cheon GJ, Paeng JC, et al. Usefulness of MRI-assisted metabolic volumetric parameters provided by simultaneous ${ }^{18} \mathrm{~F}$-fluorocholine PET/MRI for primary prostate cancer characterization. Eur J Nucl Med Mol Imaging. 2015; 42:1247-1256.

39. Jambor I, Kuisma A, Kähkönen E, et al. Prospective evaluation of ${ }^{18} \mathrm{~F}-\mathrm{FACBC}$ PET/CT and PET/MRI versus multiparametric MRI in intermediate- to high-risk prostate cancer patients (FLUCIPRO trial). Eur J Nucl Med Mol Imaging. 2018;45:355-364.

40. Lake ST, Greene KL, Westphalen AC, et al. Optimal MRI sequences for ${ }^{68} \mathrm{Ga}-$ PSMA-11 PET/MRI in evaluation of biochemically recurrent prostate cancer. EJNMMI Res. 2017;7:77.

41. Yeh R, Dercle L, Garg I, Wang ZJ, Hough DM, Goenka AH. The role of ${ }^{18} \mathrm{~F}-$ FDG PET/CT and PET/MRI in pancreatic ductal adenocarcinoma. Abdom Radiol. 2018;43:415-434.

42. Beiderwellen KJ, Poeppel TD, Hartung-Knemeyer V, et al. Simultaneous ${ }^{68} \mathrm{Ga}-$ DOTATOC PET/MRI in patients with gastroenteropancreatic neuroendocrine tumors: initial results. Invest Radiol. 2013;48:273-279.

43. Hope TA, Pampaloni MH, Nakakura E, et al. Simultaneous ${ }^{68} \mathrm{Ga}$-DOTA-TOC PET/MRI with gadoxetate disodium in patients with neuroendocrine tumor. Abdom Imaging. 2015;40:1432-1440.

44. Joo I, Lee JM, Lee DH, et al. Preoperative assessment of pancreatic cancer with FDG PET/MR imaging versus FDG PET/CT plus contrast-enhanced multidetector CT: a prospective preliminary study. Radiology. 2017;282:149-159.

45. Chen B-B, Tien Y-W, Chang M-C, et al. PET/MRI in pancreatic and periampullary cancer: correlating diffusion-weighted imaging, MR spectroscopy and glucose metabolic activity with clinical stage and prognosis. Eur J Nucl Med Mol Imaging. 2016;43:1753-1764

46. Chen B-B, Tien Y-W, Chang M-C, et al. Multiparametric PET/MR imaging biomarkers are associated with overall survival in patients with pancreatic cancer. Eur J Nucl Med Mol Imaging. 2018;45:1205-1217.

47. Sher AC, Seghers V, Paldino MJ, et al. Assessment of sequential PET/MRI in comparison with PET/CT of pediatric lymphoma: a prospective study. AJR. 2016; 206:623-631.

48. Chen KT, Gong E, de Carvalho Macruz FB, et al. Ultra-low-dose ${ }^{18} \mathrm{~F}$-florbetaben amyloid PET imaging using deep learning with multi-contrast MRI inputs. $R a$ diology. 2019;290:649-656.

49. Stecco A, Buemi F, Cassarà A, et al. Comparison of retrospective PET and MRI-DWI (PET/MRI-DWI) image fusion with PET/CT and MRI-DWI in detection of cervical and endometrial cancer lymph node metastases. Radiol Med.2016;121:537-545.
50. Beiderwellen K, Grueneisen J, Ruhlmann V, et al. [ ${ }^{18}$ F]FDG PET/MRI vs. PET/ $\mathrm{CT}$ for whole-body staging in patients with recurrent malignancies of the female pelvis: initial results. Eur J Nucl Med Mol Imaging. 2015;42:56-65.

51. Sarabhai T, Tschischka A, Stebner V, et al. Simultaneous multiparametric PET/ MRI for the assessment of therapeutic response to chemotherapy or concurrent chemoradiotherapy of cervical cancer patients: preliminary results. Clin Imaging. 2018;49:163-168.

52. Sawicki LM, Kirchner J, Grueneisen J, et al. Comparison of ${ }^{18}$ F-FDG PET/MRI and MRI alone for whole-body staging and potential impact on therapeutic management of women with suspected recurrent pelvic cancer: a follow-up study. Eur J Nucl Med Mol Imaging. 2018;45:622-629.

53. Lyng H, Malinen E. Hypoxia in cervical cancer: from biology to imaging. Clin Transl Imaging. 2017;5:373-388.

54. Pinker K, Andrzejewski P, Baltzer P, et al. Multiparametric $\left[{ }^{18} \mathrm{~F}\right]$ fluorodeoxyglucose/ $\left[{ }^{18} \mathrm{~F}\right]$ fluoromisonidazole positron emission tomography/magnetic resonance imaging of locally advanced cervical cancer for the non-invasive detection of tumor heterogeneity: a pilot study. PLoS One. 2016;11:e0155333.

55. Brandmaier P, Purz S, Bremicker K, et al. Simultaneous $\left[{ }^{18}\right.$ F]FDG-PET/MRI: correlation of apparent diffusion coefficient (ADC) and standardized uptake value (SUV) in primary and recurrent cervical cancer. PLoS One. 2015;10:e0141684.

56. Munoz C, Neji R, Cruz G, et al. Motion-corrected simultaneous cardiac positron emission tomography and coronary MR angiography with high acquisition efficiency. Magn Reson Med. 2018;79:339-350.

57. Wicks EC, Menezes LJ, Barnes A, et al. Diagnostic accuracy and prognostic value of simultaneous hybrid ${ }^{18} \mathrm{~F}$-fluorodeoxyglucose positron emission tomography/magnetic resonance imaging in cardiac sarcoidosis. Eur Heart J Cardiovasc Imaging. 2018;19:757-767.

58. Abgral R, Dweck MR, Trivieri MG, et al. Clinical utility of combined FDG-PET/ MR to assess myocardial disease. JACC Cardiovasc Imaging. 2017;10:594-597.

59. Trivieri MG, Dweck MR, Abgral R, et al. ${ }^{18} \mathrm{~F}$-sodium fluoride PET/MR for the assessment of cardiac amyloidosis. J Am Coll Cardiol. 2016;68:2712-2714.

60. Morgenstern R, Yeh R, Castano A, Maurer MS, Bokhari S. ${ }^{18}$ Fluorine sodium fluoride positron emission tomography, a potential biomarker of transthyretin cardiac amyloidosis. J Nucl Cardiol. 2018;25:1559-1567.

61. Kunze KP, Dirschinger RJ, Kossmann H, et al. Quantitative cardiovascular magnetic resonance: extracellular volume, native T1 and ${ }^{18} \mathrm{~F}-\mathrm{FDG}$ PET/CMR imaging in patients after revascularized myocardial infarction and association with markers of myocardial damage and systemic inflammation. J Cardiovasc Magn Reson. 2018;20:33.

62. Hyafil F, Schindler A, Sepp D, et al. High-risk plaque features can be detected in non-stenotic carotid plaques of patients with ischaemic stroke classified as cryptogenic using combined ${ }^{18} \mathrm{~F}$-FDG PET/MR imaging. Eur J Nucl Med Mol Imaging. 2016;43:270-279.

63. Robson PM, Dweck MR, Trivieri MG, et al. Coronary artery PET/MR imaging: feasibility, limitations, and solutions. JACC Cardiovasc Imaging. 2017;10:1103-1112.

64. Marchesseau S, Seneviratna A, Sjöholm AT, et al. Hybrid PET/CT and PET/MRI imaging of vulnerable coronary plaque and myocardial scar tissue in acute myocardial infarction. J Nucl Cardiol. 2017;83:361-11.

65. Doris MK, Rubeaux M, Pawade T, et al. Motion-corrected imaging of the aortic valve with ${ }^{18} \mathrm{~F}-\mathrm{NaF}$ PET/CT and PET/MRI: a feasibility study. J Nucl Med. 2017; 58:1811-1814.

66. von Schulthess GK, Veit-Haibach P. Workflow considerations in PET/MR imaging. J Nucl Med. 2014;55(suppl):19S-24S.

67. Grueneisen J, Sawicki LM, Schaarschmidt BM, et al. Evaluation of a fast protocol for staging lymphoma patients with integrated PET/MRI. PLoS One. 2016; 11:e0157880.

68. Kirchner J, Deuschl C, Schweiger B, et al. Imaging children suffering from lymphoma: an evaluation of different ${ }^{18} \mathrm{~F}$-FDG PET/MRI protocols compared to whole-body DW-MRI. Eur J Nucl Med Mol Imaging. 2017;44:1742-1750.

69. Sekine T, Delso G, Zeimpekis KG, et al. Reduction of ${ }^{18} \mathrm{~F}-\mathrm{FDG}$ dose in clinical PET/ MR imaging by using silicon photomultiplier detectors. Radiology. 2018;286:249-259.

70. Queiroz MA, Wollenweber SD, von Schulthess G, Delso G, Veit-Haibach P. Clinical image quality perception and its relation to NECR measurements in PET. EJNMMI Phys. 2014;1:103.

71. Sah B-R, Ghafoor S, Burger IA, et al. Feasibility of ${ }^{18} \mathrm{~F}-\mathrm{FDG}$ dose reductions in breast cancer PET/MRI. J Nucl Med. 2018;59:1817-1822.

72. Gilmore CD, Comeau CR, Alessi AM, et al. PET/MR imaging consensus paper: a joint paper by the Society of Nuclear Medicine and Molecular Imaging Technologist Section and the Section for Magnetic Resonance Technologists. J Nucl Med Technol. 2013;41:108-113.

73. Subramaniam RM, Jadvar H, Colletti PM, et al. ACR and SNMMI joint credentialing statement for PET/MRI of the body. J Nucl Med. 2017;58:1174-1176.

74. Oates ME; Diagnostic Radiology Participants of ACR/SNM Task Force II. Integrated residency training pathways of the future: diagnostic radiology, nuclear radiology, nuclear medicine, and molecular imaging. J Am Coll Radiol. 2012;9:239-244. 